University of Wollongong

Research Online

Australian Institute for Innovative Materials -

Papers

Australian Institute for Innovative Materials

$1-1-2014$

Optical and electrochemical methods for determining the effective area and charge density of conducting polymer modified electrodes for neural stimulation

Alexander R. Harris

La Trobe University, alexh@uow.edu.au

Paul Molino

University of Wollongong, pmolino@uow.edu.au

Robert M. I Kapsa

University of Wollongong, robk@uow.edu.au

Graeme M. Clarke

University of Melbourne

Antonio Paolini

La Trobe University, a.paolini@latrobe.edu.au

See next page for additional authors

Follow this and additional works at: https://ro.uow.edu.au/aiimpapers

Part of the Engineering Commons, and the Physical Sciences and Mathematics Commons

Research Online is the open access institutional repository for the University of Wollongong. For further information contact the UOW Library: research-pubs@uow.edu.au 


\title{
Optical and electrochemical methods for determining the effective area and charge density of conducting polymer modified electrodes for neural stimulation
}

\begin{abstract}
Neural stimulation is used in the cochlear implant, bionic eye, and deep brain stimulation, which involves implantation of an array of electrodes into a patient's brain. The current passed through the electrodes is used to provide sensory queues or reduce symptoms associated with movement disorders and increasingly for psychological and pain therapies. Poor control of electrode properties can lead to suboptimal performance; however, there are currently no standard methods to assess them, including the electrode area and charge density. Here we demonstrate optical and electrochemical methods for measuring these electrode properties and show the charge density is dependent on electrode geometry. This technique highlights that materials can have widely different charge densities but also large variation in performance. Measurement of charge density from an electroactive area may result in new materials and electrode geometries that improve patient outcomes and reduce side effects.
\end{abstract}

\section{Keywords}

area, effective, determining, methods, stimulation, electrochemical, neural, optical, electrodes, modified, polymer, conducting, density, charge

\author{
Disciplines \\ Engineering | Physical Sciences and Mathematics
}

\section{Publication Details}

Harris, A. R., Molino, P. J., Kapsa, R. M. I., Clarke, G. M., Paolini, A. G. \& Wallace, G. G. (2014). Optical and electrochemical methods for determining the effective area and charge density of conducting polymer modified electrodes for neural stimulation. Analytical Chemistry, 87 (1), 738-746.

\section{Authors}

Alexander R. Harris, Paul Molino, Robert M. I Kapsa, Graeme M. Clarke, Antonio Paolini, and Gordon G. Wallace 


\title{
Optical and Electrochemical Methods for Determining the Effective Area
} and Charge Density of Conducting Polymer Modified Electrodes for Neural Stimulation

\author{
Alexander R. Harris ${ }^{1}$, Paul J. Molino ${ }^{2}$, Robert M.I. Kapsa ${ }^{2,3}$, Graeme M. Clark ${ }^{4}$, Antonio G. Paolini ${ }^{5}$, \\ Gordon G. Wallace ${ }^{2}$ \\ ${ }^{1}$ School of Psychological Science, La Trobe University, Bundoora, Melbourne, Victoria, 3086, \\ Australia \\ 2 Intelligent Polymer Research Institute, University of Wollongong, Wollongong, NSW, 2522, \\ Australia \\ ${ }^{3}$ Department of Neurosciences, St Vincents Hospital, Melbourne and Department of Medicine \\ University of Melbourne, Fitzroy, Victoria, 3065, Australia \\ ${ }^{4}$ School of Engineering, University of Melbourne, Parkville, Victoria, 3010, Australia \\ 5 Health Innovations Research Institute, College of Science, Engineering and Health, RMIT \\ University, Bundoora, Victoria, 3083, Australia
}

Email: alexrharris@gmail.com 


\section{Page 2 of 24}




\begin{abstract}
Neural stimulation is used in the cochlear implant, bionic eye and deep brain stimulation which involves implantation of an array of electrodes into a patient's brain. The current passed through the electrodes is used to provide sensory queues or reduce symptoms associated with movement disorders and increasingly for psychological and pain therapies. Poor control of electrode properties can lead to sub-optimal performance; however there are currently no standard methods to assess them, including the electrode area and charge density. Here we demonstrate optical and electrochemical methods for measuring these electrode properties and show the charge density is dependent on electrode geometry. This technique highlights that materials can have widely different charge densities, but also large

variation in performance. Measurement of charge density from an electroactive area may result in new materials and electrode geometries that improve patient outcomes and reduce side-effects.
\end{abstract}

\title{
Keywords
}

Electroactive polymer; Deep Brain Stimulation; Neural prosthesis; Surface analysis; Charge Density 


\section{Introduction}

Neural stimulation can be used to provide sensory queues as in the cochlear implant and bionic eye.

Deep brain stimulation (DBS) is also being increasingly used to treat symptoms in a range of diseases, including epilepsy, Parkinson's disease, chronic pain, depression and obsessive compulsive disorder ${ }^{1}$.

For instance, the remarkable ability of DBS to reduce the severe tremor, dyskinesia and dystonia associated with Parkinson's disease can be seen in patient videos online as they switch their devices on and off. While clinical devices are being used already, the mechanism involved in DBS is still not clear $^{2}$. A deeper understanding of these devices is required to enable development of better implants and treatment regimes.

For neural stimulation, charge must be delivered from an electrode to the target neurons. One parameter that is typically used to assess an electrodes performance is charge density, the amount of charge it can deliver per unit area ${ }^{3}$. By increasing the charge density of an electrode, the electrode size can be reduced, potentially allowing stimulation of individual neurons. Furthermore, by reducing the overall device size, less surgical trauma is induced and the immune response can be minimised ${ }^{4}$.

DBS electrodes are currently all platinum, and the charge density is usually assessed by cyclic voltammetry ${ }^{5,6}$. Under this condition, the charge density is related to the capacitance current generated at the electrode-solution interface. A more detailed understanding of the charge density was achieved by measuring the current associated with adsorption of hydride in $\mathrm{HClO}_{4(\mathrm{aq})}$ or $\mathrm{H}_{2} \mathrm{SO}_{4(\mathrm{aq})}$, thus allowing the measurement of an electroactive area, rather than geometric area ${ }^{7}$. The improved method for measuring charge density lead to the application of electrode roughening steps to increase the charge available ${ }^{8}$.

More recently, there has been a rise in publications on modified electrodes using new materials to increase charge density for neural implants. Iridium oxide undergoes redox reactions to increase the charge passed during cyclic voltammetry, and hence the charge density ${ }^{5}$. Similarly, doped conducting polymers undergo a range of Faradaic and non-Faradaic reactions that result in a larger charge density ${ }^{9-14}$. More exotic materials, including carbon nanotubes, graphene, titanium nitride and

tantalum oxide have also been reported ${ }^{15-17}$. However, unlike the original platinum electrode measurements, the current methods used to obtain the charge density of these new materials and devices is not always clear. For instance, the electrode area used may be the nominal, geometric or electroactive area, but this detail is not typically reported.

This article investigates the measurement of area and charge density, including a discussion of testing solution composition, methods for measuring charge and what is meant by electrode area. To 
investigate this, commercial platinum electrode arrays were used with some of the electrodes modified with doped conducting polymers. Poly-3,4-ethylenedioxythiophene doped with 
poly(styrenesulfonate) (PEDOT-PSS) was chosen, as it has been used in many other bionics studies 18,19. para-toluene sulfonate (pTs) dopant was also tested, as previous neural recording data has shown it to have a high charge density and good acute recording performance ${ }^{20}$. Dodecylbenzenesulfonate (DBSA) dopant was also used, as it has a similar structure to pTs and had good cultured cell viability when used to dope polypyrrole ${ }^{21-23}$.

\section{Materials and Methods}

3,4-ethylenedioxythiophene (EDOT), poly(styrenesulfonate) (PSS, MW = 70,000), sodium dodecylbenzenesulfonate (NaDBSA), sodium para-toluene sulfonate (Na2pTS), hexaammineruthenium(III) chloride $\left(\mathrm{Ru}\left(\mathrm{NH}_{3}\right)_{6} \mathrm{Cl}_{3}\right)$ (Sigma-Aldrich) and $99.0 \%$ di-sodium phosphate (Fluka) were used as received. Polymer coatings were deposited on 4 shank, 32 electrode (8 electrodes per shank), $413 \mu \mathrm{m}^{2}$ nominal geometric area platinum electrodes with $200 \mu \mathrm{m}$ pitch (Neuronexus Technologies - A4x8-5mm-200-200-413). Conducting polymer coatings with different dopants were electrochemically deposited onto individual microelectrodes via a potentiostat (CH660D, CH Instruments) from mixed solutions containing $10 \mathrm{mM}$ EDOT and $0.1 \mathrm{M} \mathrm{Na}_{2}$ PTs or 2 $\mathrm{mg} \mathrm{mL}^{-1}$ NaDBSA or PSS in deionised water. Potentiostatic growth was performed in a threeelectrode configuration using one microelectrode as the working electrode, $\mathrm{Ag} / \mathrm{AgCl}(3 \mathrm{M} \mathrm{NaCl})$ as reference electrode and Pt mesh as counter electrode. Solutions were degassed for 30 minutes with nitrogen before depositing the electrode coatings. All polymers were deposited at $1 \mathrm{~V}$ vs $\mathrm{Ag} / \mathrm{AgCl}$. PEDOT-PSS and PEDOT-DBSA were deposited for 4 different times (15, 30, 45 or 60 s), PEDOTpTs was deposited for 45 s as recommended in our previous article ${ }^{20}$. 2 probes were coated with PEDOT-PSS and 2 with PEDOT-DBSA, 4 electrode sites coated at each deposition time in a staggered array as previously described ${ }^{20}$, leaving 12 uncoated platinum electrodes and 4 PEDOTpTS coated electrodes as controls.

Electrodes were imaged using a BX61 optical microscope (Olympus) and the area measured with ImageJ (figure S1). Electrochemical analysis was undertaken in $0.3 \mathrm{M}$ phosphate buffer in deionised water and the electroactive areas measured by addition of $5 \mathrm{mM} \mathrm{Ru}\left(\mathrm{NH}_{3}\right)_{6}{ }^{3+}$. Test solutions were not degassed to better represent conditions in vivo. A CHI660B potentiostat with CHI684 multiplexer (CH Instruments) were used to perform cyclic voltammetry at each of the individually addressable working electrode sites. A 3 electrode configuration was used with a Ag/AgCl (3 $\mathrm{M} \mathrm{KCl)} \mathrm{reference}$ and $\mathrm{Pt}$ mesh counter electrode. Charge density measurements were performed using cyclic voltammetry over a range of 0.8 to $-0.8 \mathrm{~V}$ vs $\mathrm{Ag} / \mathrm{AgCl}$ at a scan rate of $100 \mathrm{mV} \mathrm{s}{ }^{-1}$. Electroactive area measurements were undertaken over a range of 0 to $-0.5 \mathrm{~V}$ varying the scan rate from $10 \mathrm{mV} \mathrm{s}^{-1}$ to 1 $\mathrm{V} \mathrm{s}^{-1}$. 


\section{Results}

Electrode coatings were performed as described previously for polypyrrole (PPy) and PEDOT doped with sulphate or $\mathrm{pTs}^{20}$, except for depositing on platinum electrodes rather than iridium to prevent the formation of iridium oxide. Uncoated platinum electrodes were bright silver while PEDOT-pTs, PEDOT-PSS and PEDOT-DBSA were blue (figure S1). Consistent with previous results, PEDOTpTs uniformly coated the electrode when deposited for $45 \mathrm{~s}$. In contrast, PEDOT-PSS and PEDOTDBSA showed very rough, nodular structure growing from the electrode edge, typically with a depression over the electrode. The polymer growth was guided by the electrode wires, increasing in size with deposition time (figure S2a). Some of the conducting polymer grew around the edge of the shank, this was most likely to occur at the tip of the shank where the shank width was approximately $33 \mu \mathrm{m}$. $45 \mathrm{~s}$ deposition of PEDOT-PSS and PEDOT-DBSA produced significantly larger and rougher electrodes than 45 s deposition of PEDOT-pTs (table 1).

The charge passed during deposition of the conducting polymer increased with time due to the growth in electrode area (figure S2b). During this phase, EDOT diffuses to the electrode from in front of the shank. If the conducting polymer grew to the edge of the shank, a large increase in deposition charge was observed, leading to a series of high outliers in figure S2b. This large increase in charge is attributed to the expanded diffusion field, allowing oxidation of EDOT on the side and behind the shank. With $45 \mathrm{~s}$ deposition, PEDOT-PSS and PEDOT-DBSA had larger deposition charges than PEDOT-pTs (figure S2b). This difference is most likely due to polymer templating by the different dopant ions, increasing the polymer growth rate, rather than a change in solution properties ${ }^{24}$.

A comparison of optical area of the electrodes and the deposition charge revealed a correlation of increased area with increased deposition charge (figure S3). The electrodes with high deposition charge that deviate from this correlation all made contact with the edge of the shank. The conducting polymer on these electrodes was able grow down the side of the shank and possibly around the back. Therefore, the optically measured area for these electrodes, as viewed from above the shank, was undervalued.

Cyclic voltammetry was undertaken in $0.3 \mathrm{M}$ phosphate buffer, beginning at $0.8 \mathrm{~V}$ to $-0.8 \mathrm{~V}$ (figure S4). Bare platinum electrodes showed a reduction current beginning around $-0.1 \mathrm{~V}$ and extending down to the solvent window at $-0.8 \mathrm{~V}$, switching the potential direction, the current crosses over itself at -0.6 and again at $0 \mathrm{~V}$ (figure S4a). This process is most likely associated with the reduction of oxygen in the non-degassed solution. Voltammetry of PEDOT-pTs was consistent with previous results ${ }^{20}$, displaying a relatively featureless response with large capacitance (figure S4b). PEDOT- 
PSS possessed a broad reduction process around $-0.6 \mathrm{~V}$, shifting towards $-0.43 \mathrm{~V}$ on thicker films and

a sharper oxidation peak at $-0.37 \mathrm{~V}$ (figure S4c) ${ }^{18,19}$. The double layer capacitance was larger than 

the other coatings, around $200 \mathrm{nA}$ on the thickest film, but the potential window on the oxidation scan shifted to less positive values as the film thickness increased. PEDOT-DBSA showed a reduction peak at $-0.6 \mathrm{~V}$, shifting to $-0.51 \mathrm{~V}$ at $60 \mathrm{~s}$ deposition with a second, broader process peaking around $0.22 \mathrm{~V}$ and oxidation processes occurring from around -0.6 to $0.6 \mathrm{~V}$ (figure S4d).

The charge passed during voltammetry can be measured by transforming the current-potential response into a current-time plot and integrating the reductive or oxidative regions ${ }^{25}$. Many of the uncoated electrodes showed little or no oxidative charge over the potential window tested, and the ratio of oxidative to reductive charge on most coated electrodes was well below 1 , in contrast to our previous results ${ }^{20}$. Therefore, the reductive charge was used for all subsequent data and for calculating the charge density. A plot of the reduction charge versus deposition time reveals 3 groups, all increasing in charge with deposition time (figure S5a). The smallest charge (less than $-2 \mu \mathrm{C}$ ) is associated with the typical electrode coatings confined to the top of the shank, the group of electrodes with reduction charge between -2 and $-4 \mu \mathrm{C}$ have coatings that touch the edge of the shank while those with reduction charge greater than $-4 \mu \mathrm{C}$ are all at the tips of the shanks and grow to the shank edge. When the reduction charge is plotted against the deposition charge, a linear correlation is seen for all coatings confined to the top of the shank, with deviations for those at the tip of the shank and ones that grow to the edge (figure S5b).

For a bare electrode, the charge measured during voltammetry should be solely associated with the double layer capacitance, and therefore correlated with the electrode area. This can be described by the Helmholtz model

$$
C_{\mathrm{d}}=\varepsilon \varepsilon_{0} A / d
$$

where $C_{\mathrm{d}}$ is the capacitance, $\varepsilon$ is the dielectric constant of the solution, $\varepsilon_{0}$ is the permittivity of free space, $A$ is the electrode area and $d$ is the thickness of the double layer. More complex models of the double layer such as the Gouy-Chapman theory can also be used. However, these equations don't hold when oxygen is present, as it has an irreversible reduction process within the potential window tested, and its associated reduction current will depend on its concentration and the nature of the electrode surface. For conducting polymer modified electrodes, other Faradaic and ion transfer processes can further reduce the correlation between reductive (or oxidative) current and electrode area measured by double layer capacitance.

Clearly the measurement of electrode area optically and by reduction (or oxidation) capacitance current have limitations related to electrode roughness, growth around the shank and more complex charge transfer processes. An electroactive area will measure the total electrochemically functional electrode area including its roughness (regions that are non- or poorly conducting will not be 
measured). The adsorption of hydride on platinum electrodes has been used previously to measure the electroactive area of platinum electrodes ${ }^{7}$, however this reaction mechanism is not possible on modified electrodes. Reduction of another solution phase redox active species can be used instead. $\mathrm{Ru}\left(\mathrm{NH}_{3}\right)_{6}{ }^{3+}$ and its reduced form $\mathrm{Ru}\left(\mathrm{NH}_{3}\right)_{6}{ }^{2+}$ are highly soluble and stable in aqueous solutions, furthermore the electron transfer is outer sphere (the complex doesn’t bind to the electrode surface) and has fast kinetics. The one electron reduction

$$
\mathrm{Ru}\left(\mathrm{NH}_{3}\right)_{6}^{3+}+e^{-} \mathrm{O} \quad \mathrm{Ru}\left(\mathrm{NH}_{3}\right)_{6}^{2+}
$$

at fast scan rates, generates a peak shaped voltammetric response with a peak current according to

$$
i_{\mathrm{p}}=\left(2.69 \times 10^{5}\right) n^{3 / 2} A D^{1 / 2} C U^{1 / 2}
$$

where $n$ is the number of electrons transferred, $D$ is the diffusion coefficient $\left(9.0 \times 10^{-6} \mathrm{~cm}^{2} \mathrm{~s}^{-1}\right)^{26}, C$ is the concentration and $\mathbf{v}$ is the scan rate. At these fast scan rates (short measurement times), $\mathrm{Ru}\left(\mathrm{NH}_{3}\right)_{6}{ }^{3+}$ diffuses towards the electrode surface in a linear profile and is reduced over the areas of electrode that are electrochemically functional. The electrode area measured then includes all the crevices that $\mathrm{Ru}\left(\mathrm{NH}_{3}\right)_{6}{ }^{3+}$ can access, providing information on the electrode roughness and the uniformity of charge density over the surface. At slower scan rates (long measurement times), all of the $\mathrm{Ru}\left(\mathrm{NH}_{3}\right)_{6}$ within the crevices and between nodules is reduced and more must diffuse towards the electrode from the bulk of the solution. If the electrode is small enough or the measurement time is long enough, a sigmoidal shaped response is seen, and at a disc electrode the steady-state current $\left(i_{\mathrm{ss}}\right)$ has the form

$$
i_{\mathrm{ss}}=4 n F D C r
$$

where $F$ is the Faraday constant and $r$ is the electrode radius. A more generalised form of the equation is applied when other electrode geometries are used

$$
i_{\mathrm{ss}}=4 n F A C m
$$

where $m$ is a mass-transfer coefficient and depends on the geometry. The area measured by the reduction of $\mathrm{Ru}\left(\mathrm{NH}_{3}\right)_{6}$ is an electroactive area, and as neurons are stimulated by an electrical current, it is potentially a better guide to what a neuron would "see" than the geometric area or a mechanically measured area including roughness.

The reduction of $\mathrm{Ru}\left(\mathrm{NH}_{3}\right)_{6}{ }^{3+}$ at an uncoated electrode showed a sigmoidal response at a scan rate of $20 \mathrm{mV} \mathrm{s}^{-1}$. The steady-state voltammetry is shown after background subtraction of a voltammogram without the addition of $\mathrm{Ru}\left(\mathrm{NH}_{3}\right)_{6}{ }^{3+}$ (figure 1a). A steady-state current of approximately $10 \mathrm{nA}$ is seen with a mid-point potential of $-0.24 \mathrm{~V}$. Assuming a disc electrode geometry, this gives a steady-state electroactive area of $91 \mu^{2}$. A similar response is seen on PEDOT-pTs with a steady-state current of $17 \mathrm{nA}$, equating to a $252 \mu^{2}$ steady-state electroactive area (figure $1 \mathrm{~b}$ ). Only three electrodes coated with PEDOT-PSS displayed a steady-state response down at 10 or $20 \mathrm{mV} \mathrm{s}^{-1}$, two with $15 \mathrm{~s}$ and one at 
30 s deposition, all other PEDOT-PSS electrodes had a peak shaped voltammogram (figure 1c). This indicates the electrodes were very large and required slower scan rates (longer times) to achieve a steady-state response. In contrast, PEDOT-DBSA did reach steady-state at all deposition times tested, $i_{\mathrm{ss}}$ increasing in magnitude with deposition time (figure $1 \mathrm{~d}$ ).

The voltammetry of $\mathrm{Ru}\left(\mathrm{NH}_{3}\right)^{6^{3+}}$ at a scan rate of $200 \mathrm{mV} \mathrm{s}^{-1}$ produced peak shapes on all electrodes except $15 \mathrm{~s}$ deposition of PEDOT-PSS (figure 2). Uncoated electrodes had a reduction peak ( $\left.E_{\mathrm{p}}^{\mathrm{red}}\right)$ at $-0.3 \mathrm{~V}$ and an oxidation peak $\left(E_{\mathrm{p}}^{\mathrm{ox}}\right)$ at $-0.17 \mathrm{~V}$, giving a peak splitting $\left(\Delta E_{\mathrm{p}}=\underset{\mathrm{p}}{E_{\mathrm{p}}^{\mathrm{red}}}-E_{\mathrm{px}}^{\mathrm{ox}}\right)$ of $130 \mathrm{mV}$ and a mid-point potential $\left(E_{1 / 2}=E_{\mathrm{p}}^{\mathrm{red}}+E_{\mathrm{p}}^{\mathrm{ox}} / 2\right)$ of $-0.235 \mathrm{~V}$. While at a scan rate of $1 \mathrm{~V} \mathrm{~s}^{-1}, \Delta E_{\mathrm{p}}$ decreases to $100 \mathrm{mV}$, the peak splitting is still greater than the expected $60 \mathrm{mV}$, and suggests the diffusion profile is not completely linear. Use of scan rates above $1 \mathrm{~V} \mathrm{~s}^{-1}$ could not be performed on most electrodes as the backgroynd capacitance became too large, obscuring the current associated with the reduction of $\mathrm{Ru}\left(\mathrm{NH}_{3}\right)_{6}$. Calculation of the linear electroactive area according to equation 2 is therefore not strictly correct; however it does provide a close approximation and can be used to compare different electrodes when tested in the same manner. To minimise this error, the linear electroactive area was calculated using the fastest scan rate possible for each electrode. The uncoated electrode had a reduction peak current $\left(i_{\mathrm{p}}^{\text {red }}\right.$ ) of $12.1 \mathrm{nA}$, giving a linear electroactive area of $14.2 \mu \mathrm{m}^{2}$. PEDOT-pTs had $E_{\mathrm{p}}^{\mathrm{red}}$ of $-0.29 \mathrm{~V}, E_{\mathrm{p}}^{\mathrm{ox}}=-0.18 \mathrm{~V}$, giving $\underset{\mathrm{p}}{\Delta E_{\mathrm{p}}}=110 \mathrm{mV}$ which decreased to $90 \mathrm{mV}$ when tested at a scan rate of $1 \mathrm{~V} \mathrm{~s}^{-1}$. An $i_{\mathrm{p}}^{\text {red }}=22.2 \mathrm{nA}$ gave a linear electroactive area of $31.7 \mu^{2}$. PEDOT-PSS showed peaked voltammetry at 30, 45 and $60 \mathrm{~s}$ deposition times and had $E_{\mathrm{p}}^{\text {red }}=-0.29,-0.3$ and $-0.3 \mathrm{~V}$ respectively and $E_{\mathrm{p}}^{\mathrm{ox}}=-0.23 \mathrm{~V}$ for all times giving $\Delta E_{\mathrm{p}}$ close to $60 \mathrm{mV}$ on all electrodes. $i_{\mathrm{p}}^{\text {red }}$ increased with deposition time from 123 to 195 and 244 nA, giving linear electroactive areas of 240, 300 and $404 \mu^{2}$. Voltammetry at PEDOT-DBSA could not be background subtracted due to the large background current. $\quad E_{\mathrm{p}}^{\mathrm{red}}$ was around $-0.31 \mathrm{~V}$ at $15 \mathrm{~s}$ deposition, shifting to -0.34 at $60 \mathrm{~s}$ deposition, $E_{\mathrm{p}}^{\mathrm{ox}}$ was $-0.18 \mathrm{~V}$ at $15 \mathrm{~s}$ deposition, shifting to $-0.21 \mathrm{~V}$ at $60 \mathrm{~s}$ deposition, $\Delta E_{\mathrm{p}}$ was then close to $130 \mathrm{mV}$ for all electrodes. $i_{\mathrm{p}}^{\text {red }}$ increased with deposition time, being 22.9, 30.9, 36.9 and $49.4 \mathrm{nA}$ for 15, 30, 45 and $60 \mathrm{~s}$ deposition times and giving linear electroactive areas of 36.7, 55.1, 68.6 and $92.5 \mu \mathrm{m}^{2}$.

Both the steady-state and linear electroactive areas can be plotted against the deposition times for each polymer (figure 3). As few PEDOT-PSS electrodes obtained a steady-state response, little can be seen in figure 3a. The linear electroactive area of PEDOT-PSS reveals little correlation with 
deposition time (figure 3b). In contrast, PEDOT-DBSA shows linear increases in both steady-state and linear electroactive areas with deposition time (figures 3c and d). At $45 \mathrm{~s}$ deposition times, both PEDOT-PSS and PEDOT-DBSA had larger electroactive areas than PEDOT-pTs. Average values for steady-state and linear electroactive areas also show an increase in effective area with deposition time except for the PEDOT-PSS steady-state measurement (table 1).

Comparison of electroactive areas with optically measured areas also reveals vastly different behaviour between PEDOT-PSS and PEDOT-DBSA or PEDOT-pTs (figure 4). Once again the lack of steady-state response on PEDOT-PSS limits the data on figure 4a, while the linear electroactive area shows little correlation with optical area (figure 4b). Both steady-state and linear electroactive areas measured for PEDOT-DBSA display a linear correlation with the optically measured area (figures 4c and d). And when plotted in this way, the PEDOT-pTs, despite having smaller electrode areas at $45 \mathrm{~s}$ depositions than either PEDOT-PSS or PEDOT-DBSA, its electroactive area also strongly correlates with the optical area.

Finally, a charge density $\left(\mathrm{mC} \mathrm{cm}^{-2}\right)$ can be calculated for each electrode based on the reduction current and either the optical, steady-state or linear electroactive area. Each measure of charge density can then be compared (figure 5 and table 2). As PEDOT-PSS had limited steady-state responses, plots of its steady-state charge density with optical or linear charge density (figures 5a and e) have too few data points. The linear charge density could be plotted against the optical charge density (figure 5c), showing a large scatter. Unlike PEDOT-PSS, PEDOT-DBSA displays reasonable correlations between both electroactive charge density and optical charge density measures (figures $5 b$ and d), but not between electroactive charge densities (figure $5 f$ ).

\section{Discussion}

Charge density is a measure of the amount of charge passed by an electrode, as measured from an oxidation or reduction current, divided by the electrode area. The results above show charge can be measured by a cyclic voltammogram and the electrode area determined from optical or electrochemical methods. Depending on the method chosen, the charge density can be extremely different (table 2).

When measuring the charge for neural stimulation, there are several choices of experimental method.

The solution used should be similar to the target tissue. In this case, a simple phosphate buffer was chosen, but an argument could be made to use a more complex testing solution ${ }^{27,28}$. In particular, placement of the electrode into the body results in rapid fouling by proteins, which affect the charge measured from cyclic voltammetry ${ }^{20}$. Addition of a protein to the testing solution may therefore increase the accuracy of an electrodes acute implantation charge density, but may be of limited use for 
chronic performance when electrode encapsulation occurs ${ }^{15}$. Oxygen is also present in the body and is electroactive, therefore it should also be present in the testing solution to provide a more accurate charge measurement. Regardless of the choice in testing solution composition, it will alter the electrode capacitance and affect the charge measured. The testing solution must be consistent when testing different materials and electrode configurations to enable a true comparison.

The charge can be measured by a variety of electrochemical methods. Cyclic voltammetry has been used for testing most neural electrodes, but amperometric methods could also be used ${ }^{28}$. When using cyclic voltammetry, a choice must be made of potential window, scan rate and reduction or oxidation current. For an uncoated electrode that obeys theories such as the Helmholtz model, variation in specific electrochemical parameters have little effect, and different electrodes can still be compared.

However, for any non-ideal or more complex electrode system (ie. presence of oxygen, Faradaic charge transfer processes etc) any change in method will affect the charge measured. For instance, on an uncoated electrode, if cyclic voltammetry is performed from 0.8 to $0 \mathrm{~V}$, reduction of oxygen will be avoided, and the current associated with this process will not be measured, this would provide a different charge than if the potential window included the oxygen reduction process. Similarly, conducting polymers display a variety of charge transfer processes with different kinetics at various potentials, and changing potential window, scan rate or reduction or oxidation charge will give very different charge values. Use of amperometric techniques may be more closely related to how these electrodes will be used in the body, but similar choices must be made on the measurement parameters (oxidation or reduction current, what applied potential, how long should the current pulses be, etc). While an argument can again be made for more suitable techniques, once again consistency in technique is more critical for comparing different materials and electrode configurations. This means that due to the variation in results between techniques, comparison of data between different publications and labs may not be possible.

In general, increased conducting polymer deposition time or deposition charge produced electrodes with larger reduction charge (figure S5). However, there were deviations from the expected trends when the electrode coatings were at the tips of the shanks or touched the shank edge. The measurement of a single charge value for a material can therefore be affected by electrode geometry and care must be taken interpreting the results.

For many previous measurements of charge density, it has been unclear how the electrode area was determined. The nominal uncoated electrode area used in this article was $413 \mu \mathrm{m}^{2}$, however optical microscopy showed a maximum and minimum area over $10 \%$ different to this value. Clearly, use of the nominal area to determine charge density is not suitable. Optical measurement of the electrode area is much more accurate for planar electrodes, but was less useful for electrodes that were very 
rough, touched the edge or grew around the shank, leading to deviations from the expected trend in figure S3. Obviously growth around a shank would not be an issue for planar microelectrode arrays typically used for cell culture and cortical surface electrode arrays.

Figure S1 suggests PEDOT-PSS and PEDOT-DBSA are rougher than PEDOT-pTs, although optical microscopy is a poor measurement method for surface roughness. Previous literature has already measured the morphology and mechanical roughness of these types of conducting polymers and compared the growth of neurons on them ${ }^{9,11,29,30}$. However, when an electrode interacts with a neuron, it is unclear if and how nano/microscale roughness or porosity affects the electrodes ability to stimulate the neuron. The measurement of an electroactive area provides new information on an electrode which can be used to assess the efficacy of neural stimulation. Reduction of a solution phase redox active species allows the electroactive area to be determined. A steady-state response and corresponding electroactive area can only be obtained on small electrodes at long measurement times. Under these conditions, the $\mathrm{Ru}\left(\mathrm{NH}_{3}\right)_{6}{ }^{3+}$ within all the crevices and between nodules is already reduced and diffusion from the bulk solution produces the steady-state current. The electroactive area can be determined from equation 4 , assuming the mass-transfer coefficient $(m)$ is known. As the conducting polymer modified electrodes are non-uniform, complex geometries, and vary for every electrode, an analytical solution for $m$ is not available. Use of equation 3 therefore provides an equivalent electrode area for a disc geometry. The steady-state electroactive area for PEDOT-pTs and PEDOT-DBSA showed a good correlation with deposition time (figure 3c) and optical area (figure 4c). Deposition of PEDOT-PSS produced large, rough electrodes, with only 3 electrodes displaying a steady-state response at a scan rate of $10 \mathrm{mV} \mathrm{s}^{-1}$.

The long time scales required to achieve a steady-state response may not be applicable to the electrical stimulation of neurons, where short current pulses are normally used. The linear electroactive area measured at fast scan rates and using equation 2 may be more suitable. While the diffusion profile may not be fully linear at all the electrodes tested, the error associated with the electroactive area measurement is small and consistent across different electrodes. The linear electroactive area at PEDOT-pTs and PEDOT-DBSA modified electrodes shows a good correlation with deposition time (figure 3d) and optical area (figure 4d). In contrast, PEDOT-PSS modified electrodes didn't display any relationship between linear electroactive area and deposition time (figure 3b) or optical area (figure 4b).

In this and many other publications, dramatic differences have been seen on electrode morphology, roughness and area after deposition of conducting polymers doped with different ions ${ }^{12,31}$. The method proposed in this article doesn't measure the chemical functionality or composition of the electrode, but adds important new insights into these materials. PEDOT is an unbranched polymer 
that grows via an electrochemical reaction. After nucleation on a bare electrode, it grows out into the deposition solution, increasing the electrode area. It has been shown that typical dopant ions have minimal effect on the bulk deposition solution properties (viscosity), instead the dopant most likely interacts with the polymer growth ${ }^{24}$. Directing of polymer growth into the bulk solution essentially produces an array of microelectrodes with greater mass transport to each growth front. This would lead to increased deposition currents, forming larger and rougher electrodes for a given deposition time. Of the three dopants reported here, the larger PSS and DBSA produce larger and rougher conducting polymer growth than the small molecule pTs. Further work is required to understand this process in more detail.

Use of charge measured from cyclic voltammetry and either optical or electroactive area allows a charge density value to be determined for each electrode. For PEDOT-pTs and PEDOT-DBSA, the charge density measured from each of these effective areas has a reasonable correlation, while PEDOT-PSS appears far more variable (figure 5, table 2). The data shown here indicates careful choice of coating material and deposition time can significantly increase the charge density of an electrode. The different charge density values obtained with each area measurement method then give an indication of the electrode roughness and the uniformity of the charge density at the electrode surface.

Table 1 demonstrates all the electroactive areas are smaller than the optical areas and so the electrode area actually involved in passing charge to the solution or neuron can be significantly smaller than expected. This also implies the charge density is not uniform over an electrode surface. Recent Kelvin probe imaging has also shown non-uniform surface potentials on polypyrrole doped with hyaluronic acid ${ }^{32}$. When stimulating a neuron with a non-uniform electrode surface, the applied potential and current will be confined to the electroactive areas. This can result in a greater charge being applied to a small region and so the local charge density required to stimulated the neuron may actually be larger than expected.

It is pertinent to note that the aim of modifying electrodes is to improve the performance of electrode implants in penetrating neural applications (eg DBS) for patients and research purposes. Most of the work to increase the charge density has been for the creation of smaller electrodes that can target individual neurons or groups of neurons. However, if this modification process significantly increases the charge density coefficient of variation (table 2), it becomes difficult to separate the variation in electrode performance from the in vivo response. In particular, if highly variable electrodes are used for research purposes, it will be harder to obtain statistically significant data. PEDOT-PSS (and PPyPSS) has been one of the most widely reported conducting polymers used for neural electrode 
modification and has the largest charge density reported here. It also has a larger coefficient of variation, and for this reason, we do not recommend it for in vivo research purposes.

Current clinical devices for DBS and other bionic applications such as cochlear implants and the bionic eye have different geometries to the Neuronexus electrode array used here, with one main difference being larger electrode size. The parameters used for electrode modification described here and in previous literature will be similar on the clinical devices. The analysis technique and theory proposed in this article are also still applicable. A larger electrode will reduce the scan rate (increase the time) required to obtain a steady-state response, which may prevent observation of this type of electrochemical profile. It will also increase the background capacitance, which may obscure the $\mathrm{Ru}\left(\mathrm{NH}_{3}\right)_{6}{ }^{3+}$ voltammetry at fast scan rates, requiring slower scan rates for measuring the linear electroactive area. Different electrode geometries will also affect the diffusion profile, and subsequently the voltammetric response. Issues related to conducting polymer growth around the electrode shank may not be applicable to the clinical devices, but lateral growth, changes in surface roughness and variation in charge density will still occur and can be measured by the methods presented in this article.

While it is important to determine the most accurate measure of charge density and understand the charge transfer mechanisms involved in these neural electrodes, the correlation of charge density to neural stimulation is the ultimate test of a material. A controlled method to compare electrodes in vivo, as recently demonstrated for acute neural recording electrodes ${ }^{20,25}$, is still needed. Only then can the effect of surface roughness, electrode area and charge density be determined and the electrode design optimised.

\section{Conclusions}

Doped conducting polymers were used to modify neural implants. The charge density at each electrode was determined by measuring charge from cyclic voltammetry and electrode area optically or electrochemically. Charge passed at a modified electrode was greater than an unmodified platinum electrode, but was dependent on the testing method and electrode geometry. Optical microscopy showed the nominal electrode area to have greater than $10 \%$ error. Measurement of an electroactive area could be achieved by reduction of a redox species in solution and provide a steady-state or linear diffusion value. The electroactive areas of all the modified and unmodified electrodes were greatly smaller than the optical areas, indicating the charge density at the electrode surfaces was not uniform. The calculated charge density was greater at all modified electrodes, but it also depended on testing method and electrode geometry. Correlation between charge density values could be seen on PEDOT-pTs and PEDOT-DBSA, but not PEDOT-PSS. 


\section{Acknowledgements}

The authors acknowledge the support of the Australian Research Council through the Centre of Excellence for Electromaterials Science.

\section{Supporting Information Available}

Optical microscopy, deposition time, deposition charge, reduction charge and area comparisons, and cyclic voltammetric response. This material is available free of charge via the Internet at http://pubs.acs.org. 


\section{References}

(1) Oluigbo, C. O.; Rezai, A. R. Biomedical Engineering, IEEE Transactions on 2011, 58, 1907-1917.

(2) Perlmutter, J. S.; Mink, J. W. Annual Review of Neuroscience 2006, 29, 229.

(3) Brummer, S. B.; Turner, M. J. Bioelectrochemistry and Bioenergetics 1975, 2, 13-25.

(4) Clark, J. J.; Sandberg, S. G.; Wanat, M. J.; Gan, J. O.; Horne, E. A.; Hart, A. S.; Akers, C. A.; Parker, J.

G.; Willuhn, I.; Martinez, V.; Evans, S. B.; Stella, N.; Phillips, P. E. M. Nat Meth 2010, 7, 126-129.

(5) Beebe, X.; Rose, T. L. Biomedical Engineering, IEEE Transactions on 1988, 35, 494-495.

(6) Cogan, S. F.; Guzelian, A. A.; Agnew, W. F.; Yuen, T. G. H.; McCreery, D. B. Journal of Neuroscience Methods 2004, 137, 141-150.

(7) Brummer, S. B.; Turner, M. J. Biomedical Engineering, IEEE Transactions on 1977, BME-24, 436439.

(8) Rose, T. L.; Robblee, L. S. Biomedical Engineering, IEEE Transactions on 1990, 37, 1118-1120.

(9) Cui, X.; Hetke, J. F.; Wiler, J. A.; Anderson, D. J.; Martin, D. C. Sensors and Actuators A: Physical 2001, 93, 8-18.

(10) Baek, S.; Green, R. A.; Poole-Warren, L. A. Journal of Biomedical Materials Research Part A 2014, $102,2743-2754$.

(11) Green, R. A.; Hassarati, R. T.; Bouchinet, L.; Lee, C. S.; Cheong, G. L. M.; Yu, J. F.; Dodds, C. W.; Suaning, G. J.; Poole-Warren, L. A.; Lovell, N. H. Biomaterials 2012, 33, 5875-5886.

(12) Yang, J.; Martin, D. C. Sensors and Actuators B: Chemical 2004, 101, 133-142.

(13) Yang, J.; Martin, D. C. Sensors and Actuators A: Physical 2004, 113, 204-211.

(14) Abidian, M. R.; Martin, D. C. Advanced Functional Materials 2009, 19, 573-585.

(15) Ward, M. P.; Rajdev, P.; Ellison, C.; Irazoqui, P. P. Brain Research 2009, 1282, 183-200.

(16) Weiland, J. D.; Anderson, D. J.; Humayun, M. S. Biomedical Engineering, IEEE Transactions on 2002, 49, 1574-1579.

(17) McCreery, D.; Agnew, W.; Yuen, T.; Bullara, L. Annals of Biomedical Engineering 1988, 16, 463 481. 
(18) Yamato, H.; Ohwa, M.; Wernet, W. Journal of Electroanalytical Chemistry 1995, 397, 163-170.

(19) Bobacka, J.; Lewenstam, A.; Ivaska, A. Journal of Electroanalytical Chemistry 2000, 489, 17-27.

(20) Harris, A. R.; Morgan, S. J.; Chen, J.; Kapsa, R. M. I.; Wallace, G. G.; Paolini, A. G. Journal of Neural Engineering 2013, 10, 016004.

(21) Thompson, B. C.; Moulton, S. E.; Richardson, R. T.; Wallace, G. G. Biomaterials 2011, 32, 38223831.

(22) Barisci, J. N.; Stella, R.; Spinks, G. M.; Wallace, G. G. Electrochimica Acta 2000, 46, 519-531.

(23) Lundin, V.; Herland, A.; Berggren, M.; Jager, E. W. H.; Teixeira, A. I. PLoS ONE 2011, 6, 1-8.

(24) Lyutov, V.; Efimov, I.; Bund, A.; Tsakova, V. Electrochimica Acta 2014, 122, 21-27.

(25) Harris, A. R.; Morgan, S. J.; Wallace, G. G.; Paolini, A. G. Journal of Visualized Experiments 2014, e51084.

(26) Barsan, M. M.; Pinto, E. M.; Florescu, M.; Brett, C. M. A. Analytica Chimica Acta 2009, 635, 7178.

(27) Harris, A. R.; Zhang, J.; Cattrall, R. W.; Bond, A. M. Analytical Methods 2013, 5, 3840-3852.

(28) Cogan, S. F.; Troyk, P. R.; Ehrlich, J.; Gasbarro, C. M.; Plante, T. D. Journal of Neural Engineering 2007, 4, 79.

(29) Gelmi, A.; Ljunggren, M. K.; Rafat, M.; Jager, E. W. H. Journal of Materials Chemistry B 2014, 2, 3860-3867.

(30) Yang, J.; Martin, D. C. Journal of Materials Research 2006, 21, 1124-1132.

(31) Abidian, M. R.; Martin, D. C. Biomaterials 2008, 29, 1273-1283.

(32) Pelto, J. M.; Haimi, S. P.; Siljander, A. S.; Miettinen, S. S.; Tappura, K. M.; Higgins, M. J.; Wallace, G. G. Langmuir 2013, 29, 6099-6108. 
Table 1. Average, standard deviation and coefficient of variation of electrode area measured optically or by reduction of $\mathrm{Ru}\left(\mathrm{NH}_{3}\right)_{6}{ }^{3+}$ for a steady-state or linear electroactive area.

\begin{tabular}{lccccccccc}
\hline Polymer coating & \multicolumn{3}{c}{ Optical Area $\left(\mu \mathrm{m}^{2}\right)$} & \multicolumn{3}{c}{ Steady-state Electroactive } & \multicolumn{3}{c}{$\begin{array}{c}\text { Linear Electroactive } \\
\text { Area }\left(\mu \mathrm{m}^{2}\right)\end{array}$} \\
\cline { 2 - 11 } & Ave & SD & CV & Ave & SD & CV & Ave & SD & CV \\
\hline 15s PEDOT-PSS & 985.8 & 128.6 & 0.13 & 123.6 & 1.0 & 0.01 & 290.3 & 241.4 & 0.83 \\
30s PEDOT-PSS & 1558.0 & 79.2 & 0.05 & 833.7 & - & - & 223.11 & 68.2 & 0.31 \\
45s PEDOT-PSS & 1813.5 & 201.1 & 0.11 & - & - & - & 201.4 & 86.8 & 0.43 \\
60s PEDOT-PSS & 2245.1 & 197.7 & 0.09 & - & - & - & 321.7 & 129.7 & 0.40 \\
45s PEDOT-pTs & 710.8 & 31.6 & 0.04 & 174.1 & 89.9 & 0.52 & 20.9 & 8.7 & 0.42 \\
Uncoated & 403.5 & 24.6 & 0.06 & 119.8 & 66.8 & 0.56 & 17.2 & 7.2 & 0.42 \\
15s PEDOT-DBSA & 816.5 & 95.8 & 0.12 & 304.4 & 112.2 & 0.37 & 33.9 & 2.3 & 0.07 \\
30s PEDOT-DBSA & 1105.1 & 165.3 & 0.15 & 466.3 & 193.5 & 0.41 & 53.0 & 7.2 & 0.14 \\
45s PEDOT-DBSA & 1432.5 & 242.4 & 0.17 & 673.2 & 199.3 & 0.30 & 76.7 & 14.4 & 0.19 \\
60s PEDOT-DBSA & 1651.0 & 340.8 & 0.21 & 693.7 & 64.2 & 0.09 & 101.2 & 11.7 & 0.12 \\
45s PEDOT-pTs & 678.3 & 59.2 & 0.09 & 246.8 & 43.1 & 0.17 & 26.8 & 2.5 & 0.09 \\
Uncoated & 430.5 & 13.0 & 0.03 & 103.0 & 8.3 & 0.08 & 15.3 & 0.7 & 0.04 \\
\hline
\end{tabular}

Table 2. Average, standard deviation and coefficient of variation of electrode charge density measured from the reduction charge and optical, steady-state or linear electroactive area.

\begin{tabular}{lccccccccc}
\hline Polymer coating & \multicolumn{3}{c}{$\begin{array}{c}\text { Optical Charge Density } \\
\left(\mathrm{mC} / \mathrm{cm}^{2}\right)\end{array}$} & \multicolumn{3}{c}{$\begin{array}{c}\text { Steady-stateCharge } \\
\text { Density }\left(\mathrm{mC} / \mathrm{cm}^{2}\right)\end{array}$} & \multicolumn{3}{c}{$\begin{array}{c}\text { Linear Charge Density } \\
\left(\mathrm{mC} / \mathrm{cm}^{2}\right)\end{array}$} \\
\cline { 2 - 11 } & Ave & SD & CV & Ave & SD & CV & Ave & SD & CV \\
\hline 15s PEDOT-PSS & 121.4 & 222.9 & 1.84 & 189.4 & 13.6 & 0.07 & 859.5 & 883.6 & 1.03 \\
30s PEDOT-PSS & 104.6 & 86.3 & 0.82 & 67.3 & - & - & 645.0 & 448.9 & 0.70 \\
45s PEDOT-PSS & 111.3 & 124.5 & 1.12 & - & - & - & 669.0 & 203.1 & 0.30 \\
60s PEDOT-PSS & 130.4 & 77.8 & 0.60 & - & - & - & 1064.7 & 555.3 & 0.52 \\
45s PEDOT-pTs & 18.6 & 0.9 & 0.05 & 102.5 & 65.2 & 0.64 & 753.1 & 353.7 & 0.47 \\
Uncoated & 15.8 & 1.9 & 0.12 & 64.6 & 21.9 & 0.34 & 415.8 & 109.5 & 0.26 \\
15s PEDOT-DBSA & 49.5 & 67.1 & 1.36 & 66.9 & 7.9 & 0.12 & 601.8 & 246.6 & 0.41 \\
30s PEDOT-DBSA & 95.1 & 121.3 & 1.28 & 78.3 & 19.7 & 0.25 & 637.7 & 73.4 & 0.12 \\
45s PEDOT-DBSA & 42.8 & 9.3 & 0.22 & 84 & 18.7 & 0.22 & 715.3 & 128.8 & 0.18 \\
60s PEDOT-DBSA & 76.1 & 40.4 & 0.53 & 110.4 & 9.8 & 0.09 & 759.1 & 83.1 & 0.11 \\
45s PEDOT-pTs & 16.5 & 0.8 & 0.05 & 46.2 & 6.8 & 0.15 & 419.1 & 43.5 & 0.10 \\
Uncoated & 14.2 & 2.0 & 0.14 & 61.2 & 5.0 & 0.08 & 410.7 & 25.3 & 0.06 \\
\hline
\end{tabular}




\section{Figure Captions}

Figure 1. Background subtracted cyclic voltammetry of $5 \mathrm{mM} \mathrm{Ru}\left(\mathrm{NH}_{3}\right)_{6}{ }^{3+}$ in $0.3 \mathrm{M} \mathrm{Na}_{2} \mathrm{HPO}_{4}$ at 10 $\mathrm{mV} \mathrm{s}^{-1}$ at (a) an uncoated electrode; and electrodes coated with (b) 45 s deposition of PEDOT-pTs (c) PEDOT-PSS (reductive scan) and (d) PEDOT-DBSA (reductive scan) at varying deposition times.

Figure 2. Background subtracted cyclic voltammetry of $5 \mathrm{mM} \mathrm{Ru}\left(\mathrm{NH}_{3}\right)_{6}{ }^{3+}$ in $0.3 \mathrm{M} \mathrm{Na}_{2} \mathrm{HPO}_{4}$ at 200 $\mathrm{mV} \mathrm{s}^{-1}$ at (a) an uncoated electrode; and electrodes coated with (b) 45 s deposition of PEDOT-pTs (c) PEDOT-PSS and (d) PEDOT-DBSA (without background subtraction) at varying deposition times.

Figure 3. Comparison of electrochemically measured electrode area versus deposition time of PEDOT doped with (a-b) PSS or (c-d) DBSA. (a and c) Steady state, (b and d) linear diffusion response of $5 \mathrm{mM} \mathrm{Ru}\left(\mathrm{NH}_{3}\right)_{6}$ in $0.3 \mathrm{M} \mathrm{Na}_{2} \mathrm{HPO}_{4}$.

Figure 4. Comparison of electrochemically measured versus optically measured electrode area (a-b) PEDOT-PSS or (c-d) PEDOT-DBSA. (a and c) Steady state, (b and d) linear diffusion response of 5 $\mathrm{mMRu}\left(\mathrm{NH}_{3}\right)_{6}$ in $0.3 \mathrm{M} \mathrm{Na}_{2} \mathrm{HPO}_{4}$.

Figure 5. Comparison of charge density with different electrode area measurements (a, c, e) PEDOTPSS (b, d, f) PEDOT-DBSA. 
a

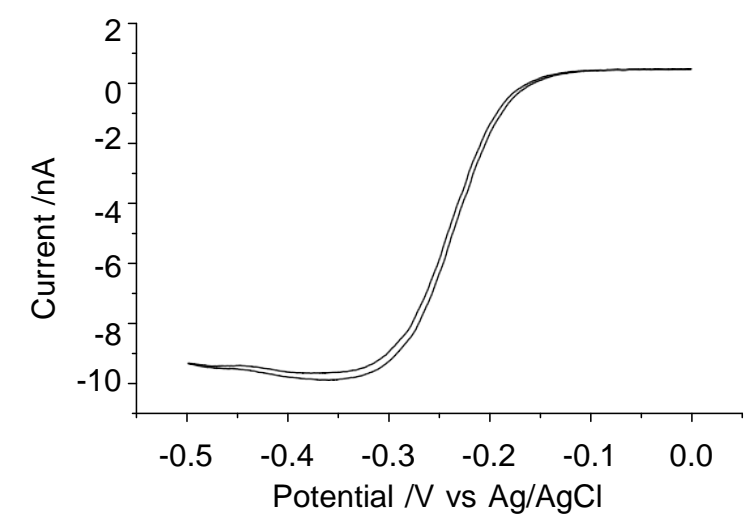

C

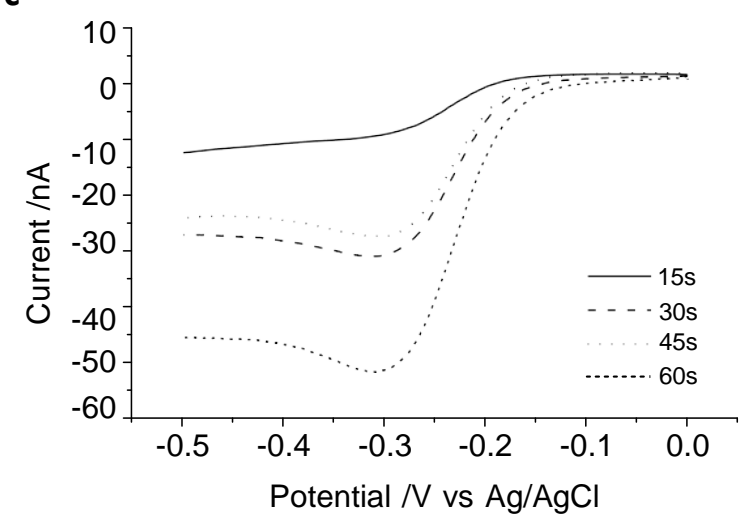

b

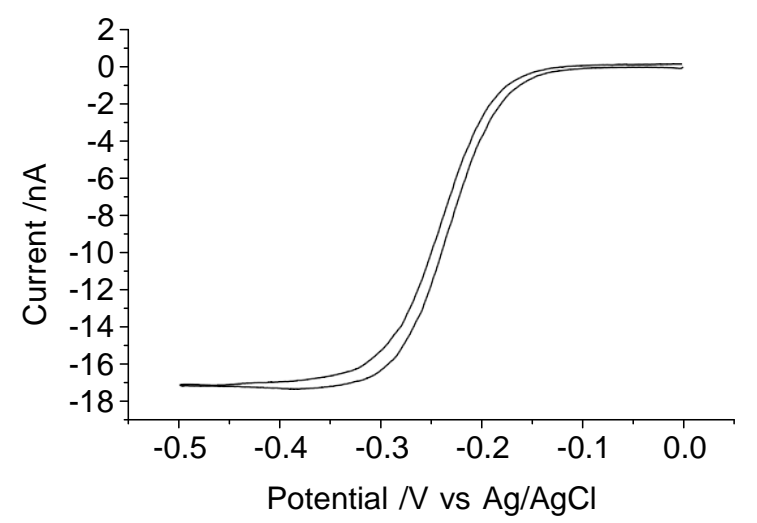

d

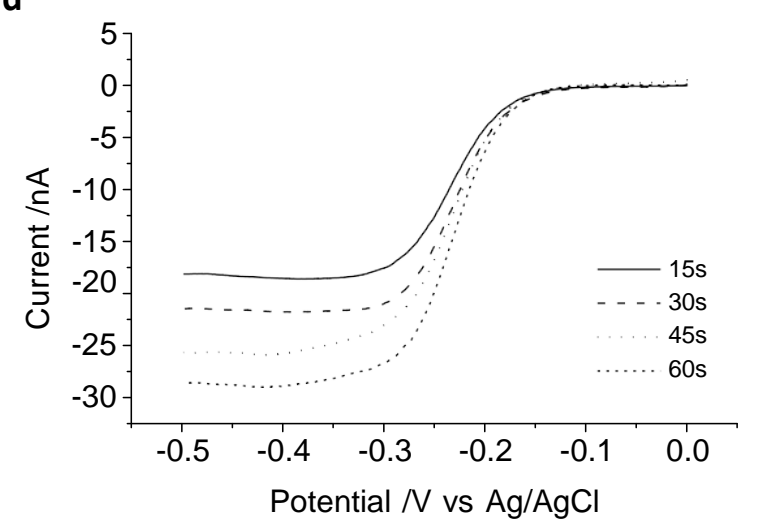

\section{Figure 1}


a

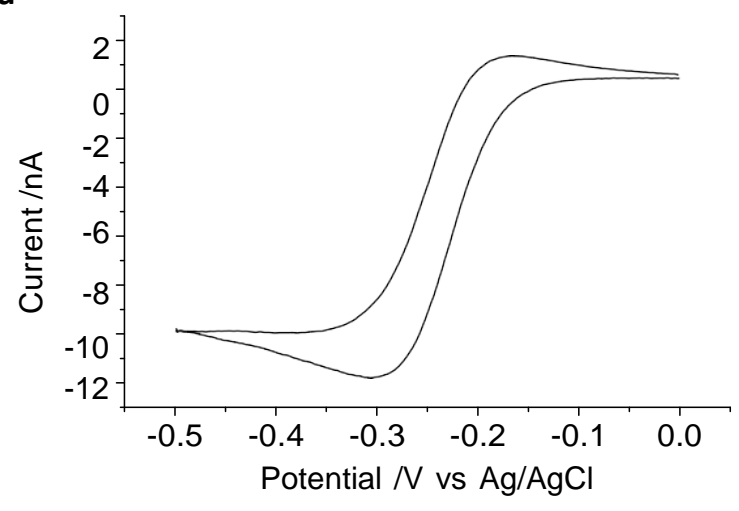

C

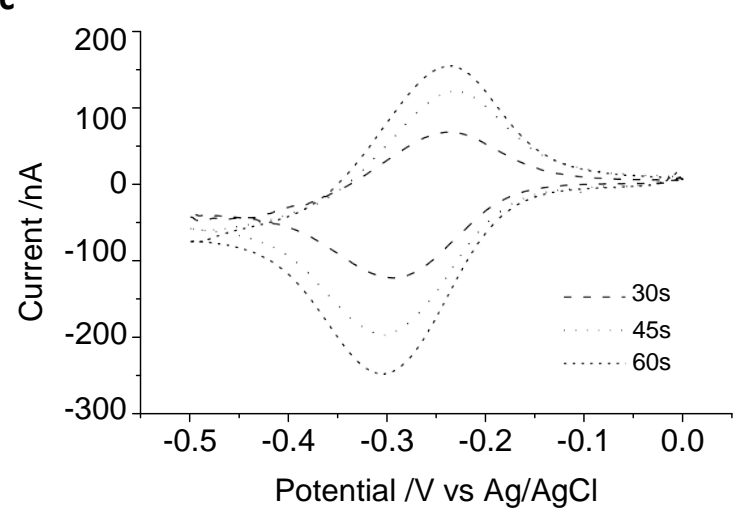

b

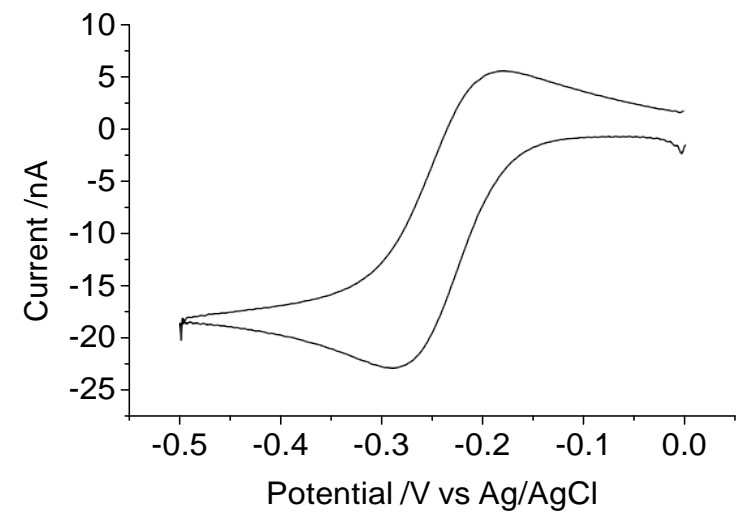

d

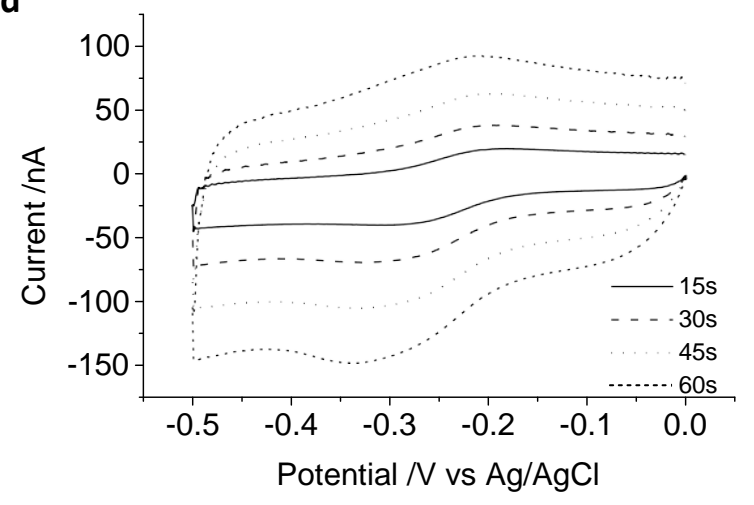

Figure 2 

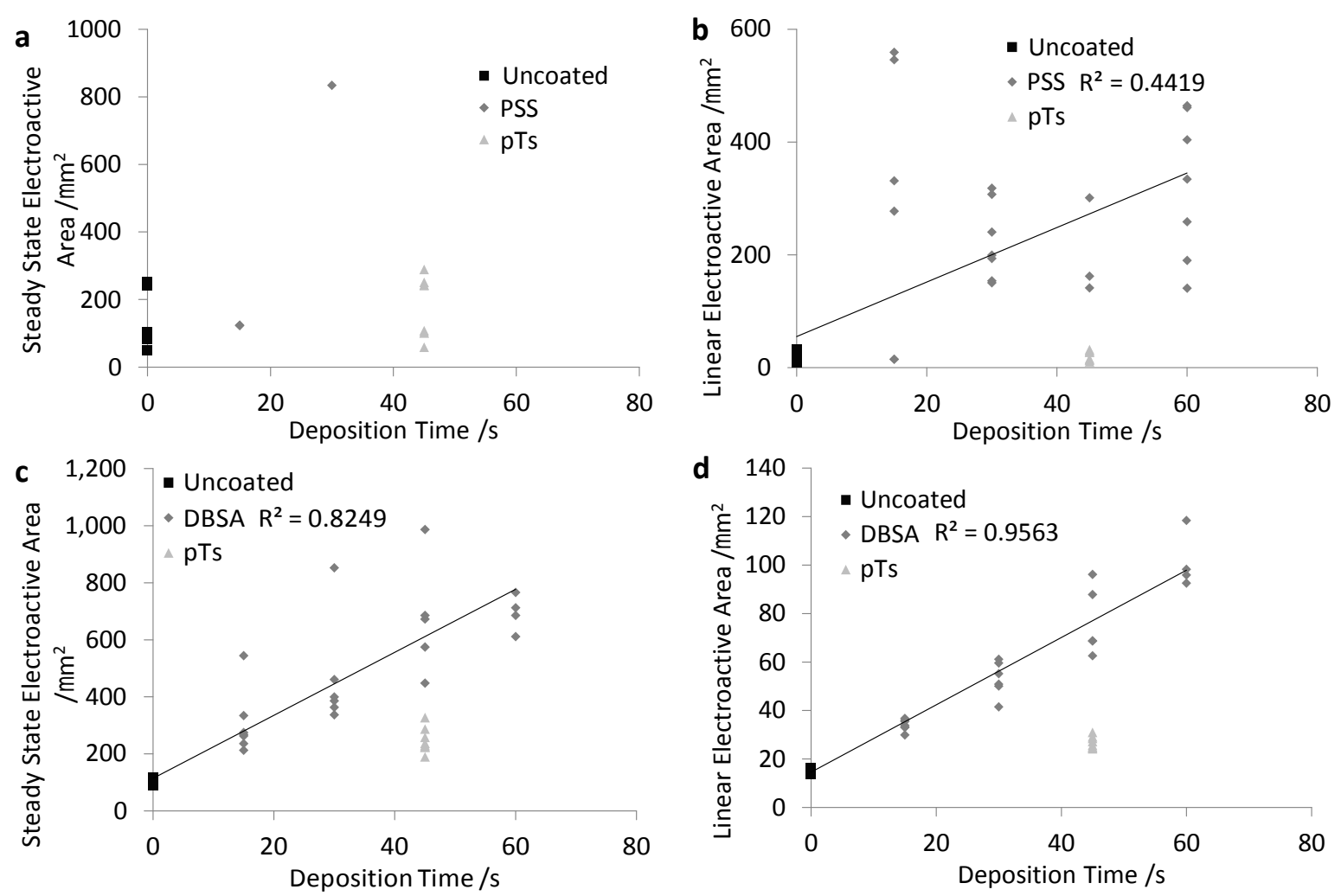

Figure 3 

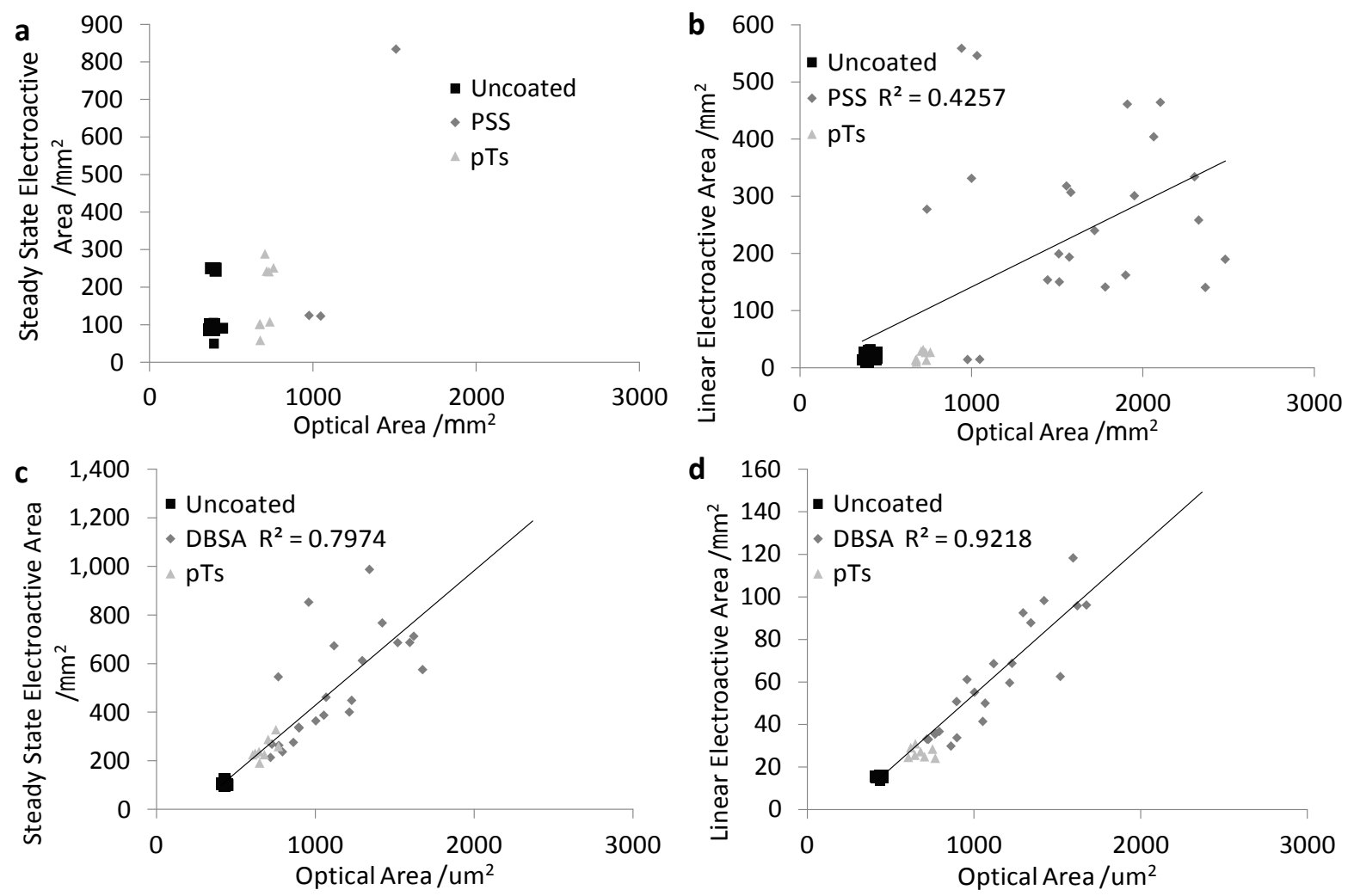

Figure 4 


\section{Page 23 of 24}
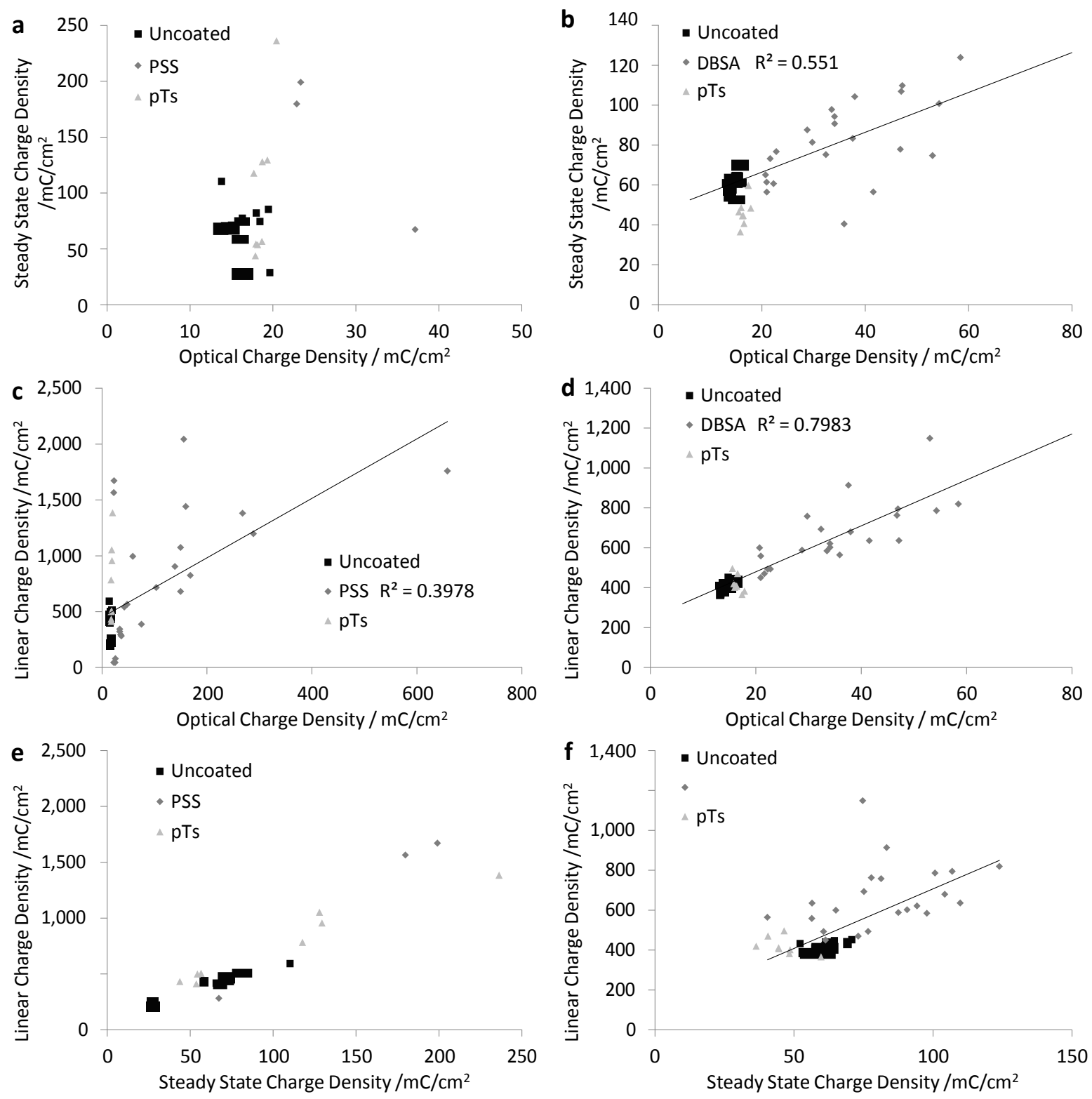

Figure 5 


\section{Page 24 of 24}

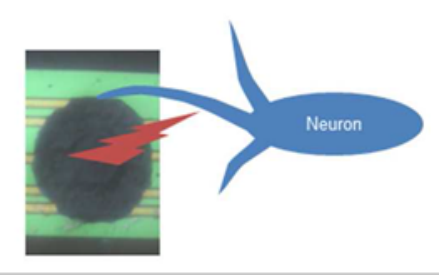

For TOC Only

$254 \times 190 \mathrm{~mm}(96 \times 96 \mathrm{DPI})$ 